\title{
Corrigendum: Left temporal alpha-band activity reflects single word intelligibility
}

\author{
Robert Becker ${ }^{1}$,Maria Pefkou' ${ }^{2}$ Christoph M. Michel ${ }^{1}$ and Alexis G. Hervais-Adelman ${ }^{2 *}$ \\ ${ }^{\prime}$ Functional Brain Mapping Lab, Department of Fundamental Neuroscience, University of Geneva, University Medical School, Geneva, Switzerland \\ ${ }^{2}$ Brain and Languge Lab, Department of Clinical Neuroscience, University of Geneva, University Medical School, Geneva, Switzerland \\ *Correspondence: alexis.adelman@unige.ch
}

Edited and reviewed by:

Jonathan E. Peelle, Washington University in St. Louis, USA

Keywords: speech intelligibility, degraded speech, noise-vocoding, alpha oscillations, left inferior temporal cortex

\section{A commentary on}

Left temporal alpha-band activity reflects single word intelligibility

by Becker, R., Pefkou, M., Michel, C. M., and Hervais-Adelman, A. G. (2013). Front. Syst. Neurosci. 7:121. doi: 10.3389/fnsys.2013.00121

Figure 5 of the article by Becker et al. (2013) contained a minor error, which we hereby rectify. In the original figure at the bottom left of panel $\mathrm{C}$ the indication of the sagittal section used for display of the inverse solution is incorrect. We therefore re-submit Figure 5 with the correct crosssection for subpanel C.

\section{REFERENCES}

Becker, R., Pefkou, M., Michel, C. M., and HervaisAdelman, A. G. (2013). Left temporal alphaband activity reflects single word intelligibility. Front. Syst. Neurosci. 7:121. doi: 10.3389/fnsys. 2013.00121

Loftus, G. R., and Masson, M. E. J. (1994). Using confidence intervals in within-subjects designs. Psychon. Bull. Rev. 1, 476-490. doi: 10.3758/ bf03210951

Received: 27 February 2014; accepted: 14 March 2014; published online: 01 April 2014.
Citation: Becker R, Pefkou M, Michel CM and Hervais-Adelman AG (2014) Corrigendum: Left temporal alpha-band activity reflects single word intelligibility. Front. Syst. Neurosci. 8:47. doi: 10.3389/fnsys. 2014.00047

This article was submitted to the journal Frontiers in Systems Neuroscience.

Copyright () 2014 Becker, Pefkou, Michel and HervaisAdelman. This is an open-access article distributed under the terms of the Creative Commons Attribution License (CC BY). The use, distribution or reproduction in other forums is permitted, provided the original author $(s)$ or licensor are credited and that the original publication in this journal is cited, in accordance with accepted academic practice. No use, distribution or reproduction is permitted which does not comply with these terms.

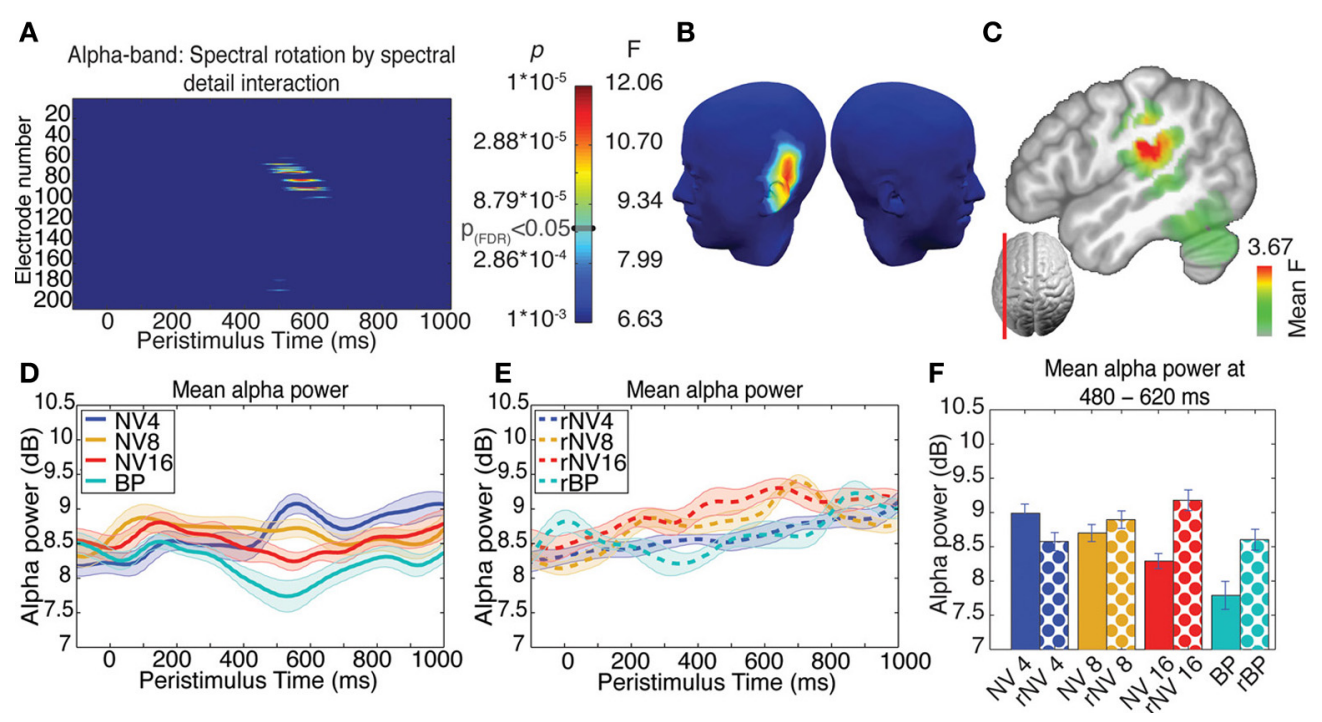

FIGURE 5 | Results of ANOVA of induced activity in the alpha-band. (A) Electrode-by-time plot of the $p$-values for the interaction of rotation $x$ spectral detail with corresponding $F$-and $p$-values, thresholded at $p=0.001$, revealing the time-window of interest $(462-633 \mathrm{~ms})$. The color bar indicates the corresponding $F$ - and $p$-values, the threshold for $p($ FDR $<0.05$ is indicated. (B) Topography of this effect, using the same color scale as in (A) at the peak of the effect (533 ms), indicating a contribution of left-temporal sources. (C) Localization of this effect in the inverse space, the main source being in the left supramarginal gyrus extending into left inferior parietal and superior temporal structures, showing the average F-statistic over the time-window of interest. (D) Average time-course of this effect in a cluster of five contributing electrodes across NV conditions, demonstrating enhanced alpha-band suppression for more intelligible conditions. (E) Corresponding time-courses for the spectrally rotated conditions, where the effect of spectral detail is absent. (F) Alpha-band activity for each condition in the significant time-window, error bars represent standard error of the mean corrected to be appropriate for repeated-measures comparisons, as described in Loftus and Masson (1994). 